\title{
ARTICLE Metabolic activity in subcallosal cingulate predicts response to deep brain stimulation for depression
}

\author{
Elliot C. Brown ${ }^{1,2,3,4,5}$, Darren L. Clark ${ }^{1,2,3,4}$, Nils D. Forkert ${ }^{2,3,6}{ }^{\text {, Christine P. Molnar }}{ }^{6}$, Zelma H. T. Kiss ${ }^{1,2,3,4}$ and \\ Rajamannar Ramasubbu (D) ${ }^{1,2,3,4}$
}

Subcallosal cingulate (SCC) deep brain stimulation (DBS) is a promising therapy for treatment-resistant depression (TRD), but response rates in open-label studies were not replicated in a large multicenter trial. Identifying biomarkers of response could improve patient selection and outcomes. We examined SCC metabolic activity as both a predictor and marker of SCC DBS treatment response. Brain glucose metabolism (CMRGlu) was measured with [18F] FDG-PET at baseline and 6 months post DBS in 20 TRD patients in a double-blind randomized controlled trial where two stimulation types (long pulse width (LPW) $n=9$ and short pulse width (SPW) $n=11$ ) were used. Responders $(n=10)$ were defined by a $\geq 48 \%$ reduction in Hamilton Depression Rating Scale scores after 6 months. The response rates were similar with five responders in each stimulation group: LPW (55.6\%) and SPW (44.5\%). First, differences in SCC CMRGlu in responders and non-responders were compared at baseline. Then machine learning analysis was performed with a leave-one-out cross-validation using a Gaussian naive Bayes classifier to test whether baseline CMRGlu in SCC could categorize responders. Finally, we compared 6-month change in metabolic activity with change in depression severity. All analyses were controlled for age. Baseline SCC CMRGlu was significantly higher in responders than non-responders. The machine learning analysis predicted response with 80\% accuracy. Furthermore, reduction in SCC CMRGlu 6 months post DBS correlated with symptom improvement $(r(17)=0.509 ; p=0.031)$. This is the first evidence of an image-based treatment selection biomarker that predicts SCC DBS response. Future studies could utilize SCC metabolic activity for prospective patient selection.

Neuropsychopharmacology (2020) 45:1681-1688; https://doi.org/10.1038/s41386-020-0745-5

\section{INTRODUCTION}

The use of deep brain stimulation (DBS) for treating psychiatric disorders such as major depressive disorder (MDD) has become a viable and relatively safe therapeutic option for people with treatment resistance. The subcallosal cingulate cortex (SCC) is the most frequent DBS target used for depression in the published literature, with success rates ranging from 30 to $80 \%$ in open-label trials at 6 months [1-6]. Yet, the only double-blind randomized, sham-controlled trial of SCC DBS found a relatively low response rate of $17 \%$ in the active stimulation condition, which was not statistically different to the sham condition [7]. This outcome implies a great need for optimization of procedures, especially in terms of the selection of patients most likely to benefit from this treatment. Previous studies have documented the potential of brain imaging markers in the prediction of antidepressant outcome. In fact, the SCC target was developed based on such an image-based biomarker [1]. However, we are yet to find an evidence-based method for patient selection that could potentially enhance clinical outcomes and minimize the risks of exposing likely non-responders to invasive surgery.

The SCC consists of mainly Brodmann area 25, which has extensive connectivity to cortical and limbic areas involved in emotional regulation. It plays a crucial role in the currently accepted neural model of depression $[8,9]$. Numerous functional imaging studies have shown hyperactivation and increased blood flow within the SCC in MDD [10-13]. Furthermore, activity in the SCC has emerged as a promising marker for predicting treatment response across multiple treatment modalities, including pharmacological, cognitive, repetitive transcranial magnetic stimulation (rTMS), electroconvulsive and sleep deprivation therapy [1, 14-21].

The first SCC DBS open-label study used positron emission tomography (PET) imaging to reveal that after 3 and 6 months of chronic DBS, three responders exhibited decreases in cerebral blood flow (CBF) in SCC, hypothalamus, anterior insula, medial frontal cortex and orbitofrontal cortex (OFC), with increases in dorsolateral prefrontal and dorsal anterior cingulate cortex [1]. A subsequent study by the same group revealed an increase in cerebral metabolic rates of glucose in SCC white matter, along with other areas, and decreases in surrounding grey matter, all 6 months post DBS $(n=8)$ [17]. Further evidence for DBS-induced decreases in SCC grey matter activity also came from another recent study involving a small number of patients receiving SCC DBS for treating refractory depression [22]. Interestingly, six patients given SCC DBS for the treatment of anorexia nervosa also showed a decrease in SCC metabolic activity after 6 and 12 months of stimulation, which was related to the amelioration of

\footnotetext{
${ }^{1}$ Mathison Centre for Mental Health Research \& Education, University of Calgary, Calgary, AB, Canada; ${ }^{2}$ Hotchkiss Brain Institute, University of Calgary, Calgary, AB, Canada; ${ }^{3}$ Clinical Neurosciences, University of Calgary, Calgary, AB, Canada; ${ }^{4}$ Department of Psychiatry, University of Calgary, Calgary, AB, Canada; ${ }^{5}$ Charité-Universitätsmedizin Berlin,

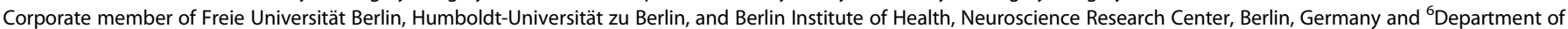
Radiology, University of Calgary, Calgary, AB, Canada

Correspondence: Rajamannar Ramasubbu (rramasub@ucalgary.ca)
}

Received: 23 November 2019 Revised: 6 May 2020 Accepted: 12 June 2020

Published online: 24 June 2020 
depressive symptoms [23]. In addition, several other DBS studies aimed at treating depression and OCD by targeting other brain areas including nucleus accumbens, ventral capsule/ventral striatum, bed nucleus of the stria terminalis and subthalamic nucleus all showed decreases in SCC activity and blood flow following DBS [24-28]. In summary, the PET findings from DBS studies targeting SCC have consistently shown DBS-related inhibition in SCC grey matter and a mixture of inhibition and activation in distal areas outside of the SCC. Furthermore, DBSinduced SCC inhibition seems to be specifically related to a reduction in depressive pathology.

However, previous SCC DBS studies reporting PET findings have used rather small sample sizes $[1,17,29]$. The unbalanced distribution of responders and non-responders in some of these studies may have further limited the possibility of using baseline brain activity to predict treatment outcome [17]. Given the paucity of data on response prediction of SCC DBS, we aimed to investigate metabolic activity, specifically in the SCC, as a candidate biomarker for predicting DBS treatment outcome and also as a marker for tracking clinical improvement in a relatively large sample size $(n=20)$. Therefore, we measured metabolic activity using $\left({ }^{18} \mathrm{~F}\right)$ Fluorodeoxyglucose PET (FDG-PET) at the preoperative baseline, and again after 6 months of chronic DBS stimulation. We hypothesized that baseline SCC metabolic activity would differentiate responders from nonresponders after 6 months of chronic DBS, and that the level of baseline SCC metabolic activity would predict the level of clinical response. Our second hypothesis was that chronic DBS would decrease SCC metabolic activity, with the degree of reduction correlating with clinical improvement.

\section{METHODS}

\section{Participants}

Twenty patients who met the DSM-IV criteria for MDD and bipolar depression with treatment resistance, and participated in our SCC DBS clinical trial, were included in this study. More information on recruitment and inclusion/exclusion criteria is provided in Supplementary Methods and in our clinical outcome paper [30]. Two patients from the original study were not included in this study because one patient died by suicide in the 3rd month so baseline PET imaging was excluded from analysis, and follow-up PET could not be performed in the second patient. Clinical response was defined as a $48 \%$ reduction in Hamilton Depression Rating Scale (HDRS) scores from baseline to 6 months postoperatively following continuous bilateral SCC DBS. While we had originally defined response as a $50 \%$ reduction in HDRS from baseline, as the study progressed, we found that this strict cutoff at a single time point could introduce misclassification in some patients. For example, half point scores cannot be used in the HDRS. Others have also highlighted how a $50 \%$ response criteria at predefined time points is misleading in DBS trials for treatment-resistant depression (TRD) with small samples [31-33]. Furthermore, depression scores seem to fluctuate between 45 and $55 \%$ in the maintenance phase of DBS treatment for TRD [33]. Specifically in our study, two subjects were categorized as responders at 6 months with a $48 \%$ reduction in HDRS from baseline. One patient showed a reduction in HDRS from a baseline of 25 to 13 at 6 months and the second subject showed reduction in HDRS from 27 to 14 . Both patients demonstrated gradual progressive decline in scores over the first 6 months. We therefore decided to change the response criterion of $48 \%$ reduction from baseline with consistent reduction in HDRS scores over time, could best differentiate responders. One patient who had a sudden $50 \%$ reduction in HDRS at 6-month visit was identified as spurious by the patient and spouse as his depression worsened a week later. Although he was categorized as responder per protocol, he was clinically considered as non-responder and crossed over to different stimulation. In this PET study he was classified as nonresponder.

Table 1 shows the demographic and clinical data of responders and non-responders. Ethical approval was given by the Institutional Review Board of the University of Calgary, the Conjoint Health Research Ethics Board. All participants provided written informed consent and the trial was registered with ClinicalTrials. gov (\# NCT01983904).

\section{DBS treatment protocol}

Briefly described, DBS electrodes were implanted into SCC white matter bilaterally using a stereotactic frame, where the target point and trajectory were defined using stereotactic 1.5 Tesla MR imaging and guided by the position of target white-matter pathways, using diffusion-based tractography. Further details about white-matter targeting, DBS programming, stimulation optimization and clinical management are provided in our clinical outcome paper [30]. A shortened CONSORT diagram of this trial is illustrated in Fig. 1. A more detailed CONSORT diagram is included in the main clinical paper [30].

Imaging data acquisition and preprocessing

Static FDG-PET scans were acquired from all patients, at baseline within 1 month preoperatively, and again after 6 months of continuous stimulation. FDG-PET measures cerebral metabolic rate of glucose utilization (CMRGlu). For all postoperative PET scans, DBS remained turned on. All patients also had high-resolution structural T1-weighted MRI taken preoperatively, which were used for image preprocessing and as an anatomical reference for the PET analysis. Details of image data acquisition and preprocessing can be found in the Supplementary Materials.

Table 1. Demographics and symptom severity scores-means, standard deviations and results of statistical tests.

\begin{tabular}{|c|c|c|c|c|}
\hline & Responders $(n=10)$ & Non-responders $(n=10)$ & Test & $p$ \\
\hline Age & $39.5 \pm 13.0$ & $54.3 \pm 12.9$ & $t=2.56$ & 0.020 \\
\hline Male/female & $4 / 6$ & $8 / 2$ & $x^{2}=3.33$ & 0.068 \\
\hline Duration of current episode (months) & $23.7 \pm 22.6$ & $26.6 \pm 22.9$ & $t=0.29$ & 0.779 \\
\hline HDRS-baseline & $23.7 \pm 4.7$ & $22.8 \pm 4.2$ & $t=-0.45$ & 0.658 \\
\hline Antidepressants at time of implant & $2.1 \pm 1.5$ & $2.1 \pm 1.2$ & $t=0.00$ & 1.000 \\
\hline
\end{tabular}




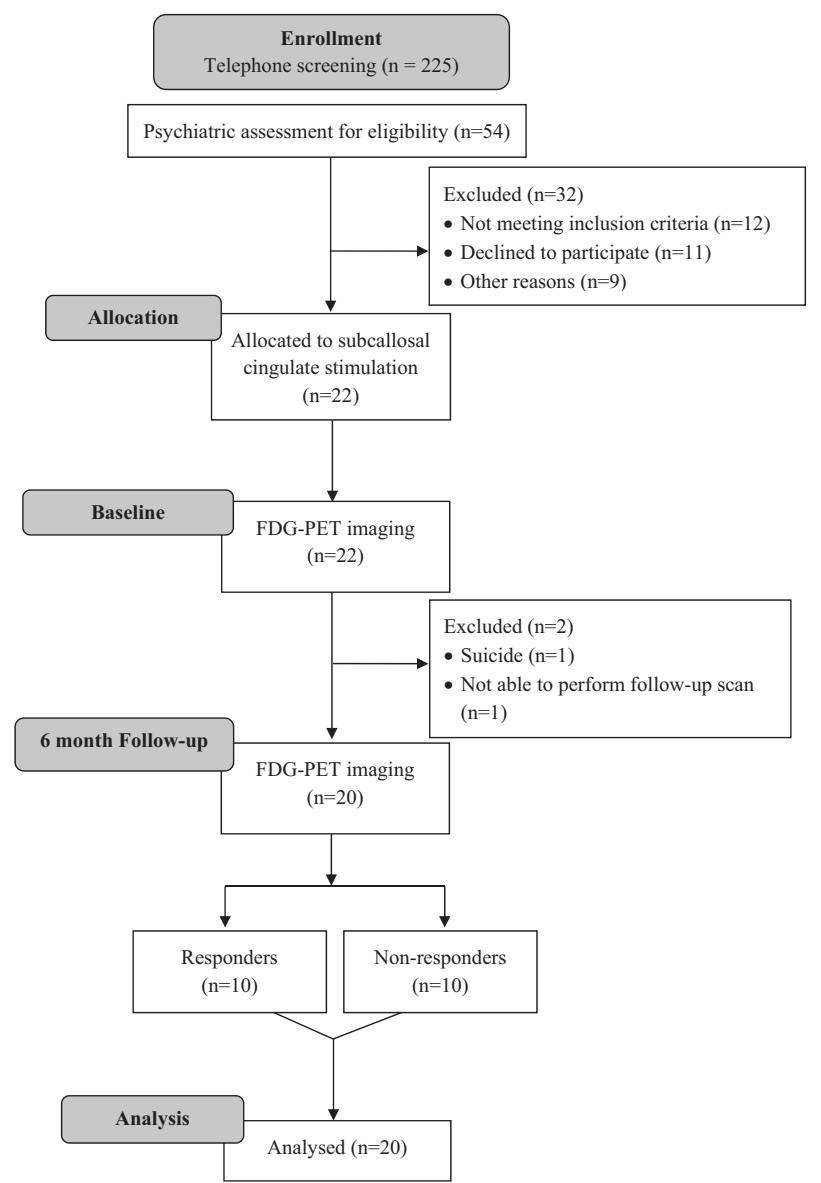

Fig. 1 CONSORT diagram. This flow diagram displays the progress of the participants included in the FDG-PET study through phases of the Subcallosal Deep Brain Stimulation trial. FDG-PET: $\left({ }^{18} \mathrm{~F}\right)$ Fluorodeoxyglucose-Positron Emission Tomography.

Imaging data analysis

A bilateral SCC region (volume) of interest (ROI) was defined anatomically using the Harvard-Oxford Atlas [34-37], labelled in the atlas as the subcallosal cortex. A binary mask of the SCC anatomical region was created at $100 \%$ probability, which made up a volume that was $91 \times 109 \times 91$ voxels, with each voxel being $2 \times 2 \times 2 \mathrm{~mm}$. The SCC ROI binary mask was used for all ROI analyses; SPM12 software (Statistical Parametric Mapping, Wellcome Centre for Human Neuroimaging, University College, London, UK) was used for all imaging analyses. Small volume correction was applied in SPM12 to voxel-wise ROI analyses and results were only considered to be statistically significant at a family-wise error (FWE) corrected $p<0.05$. In addition to the voxelwise ROI analysis, MarsBaR, an ROI toolbox for SPM [38] was used to extract mean CMRGlu values from the SCC ROI using the same binary mask in each participant. To determine whether depression pathology related to metabolic activity in the SCC, we performed a voxel-wise multiple regression within the SCC ROI using baseline FDG-PET scans, and baseline HDRS scores as a regressor of interest.

In order to determine whether baseline FDG-PET could classify response outcome, voxel-wise contrasts were performed between responders and non-responders to compare baseline metabolic activity within the SCC ROI. Age was included as a regressor of no interest, as age was significantly different between responders and non-responders at the 6-month time point. We also checked for grey matter volume group differences in the SCC (voxel-based morphometry analysis [39]), but did not find any, so we did not include grey matter volume in further analyses. To assess whether baseline metabolic activity could serve as a predictor of change in symptom severity, a voxel-wise multiple regression within the SCC ROI was performed with baseline FDG-PET scans from all patients, using 6-month percentage change in HDRS as a regressor of interest, and age as a regressor of no interest.

As a secondary analysis, we investigated whether a simple machine learning model using baseline mean CMRGlu in the SCC was capable of classifying response in individual patients. More precisely, a Gaussian naive Bayes classifier was trained and tested using the average FDG-PET signal intensity in the SCC ROI and known ground truth data (responder vs. non-responder) for the 20 patient cases. As only one feature was used for training and testing, the naive Bayes classifier essentially models the two classes (responders vs. non-responders) as Gaussian distributions, which are then used to identify the optimal threshold within the training stage and used for classification. A leave-one-out crossvalidation scheme was used for evaluation. In doing so, 19 patients were used for training of the model and the remaining patient was used for testing. By iteratively repeating this procedure for all patients, a classification was generated for each patient, which was then compared to the ground truth classification (responder vs. non-responder). Due to the rather small sample size, no completely separate validation set was available.

To address the second hypothesis of whether FDG-PET could serve as a treatment marker, i.e. whether change in symptoms related to change in SCC metabolism, a partial correlation analysis was performed between 6-month percentage change in mean CMRGlu values in the SCC ROI, and HDRS 6-month percentage change, controlling for age. As a further exploratory analysis, a voxel-wise repeated-measures ANCOVA (rmANCOVA) was applied to baseline preoperative and 6-month postoperative FDG-PET images within the SCC ROI, with time (baseline, 6 months postoperative) as the within-subjects factor for responders and non-responders separately, again using age as a regressor of no interest.

To investigate potential confounding effects of illness chronicity on treatment marker results, partial correlations were calculated between the 6-month percentage change in mean CMRGlu in SCC, and duration of illness plus duration of current episode, after controlling for age.

Finally, voxel-wise analyses of the whole brain were used to identify areas associated with baseline treatment prediction, and areas that may serve as additional potential treatment markers. All voxel-wise analyses described above were performed again investigating the whole brain. Only results at an FWE corrected $p<0.05$ at the cluster level were considered statistically significant. Given the small sample size, and exploratory nature of this analysis, we also reported results not corrected for multiple comparisons but with a minimum cluster size of ten voxels at $p<$ 0.005 (uncorrected) to identify potentially interesting regions for future research.

\section{RESULTS}

Clinical and demographic results

Among 20 patients included in the study, $16(80 \%)$ had the diagnosis of MDD and $4(20 \%)$ had the diagnosis of bipolar disorder (BD). Mean age of participants was 46.9 years $(S D=14.7)$, ranging from 22 to 69 years. Of the 20 patients, 11 (55\%) were randomized to short pulse width (SPW) and 9 patients (45\%) to long pulse width (LPW) stimulation. Following 6 months of chronic DBS, 8 of the 20 patients showed a $50 \%$ or greater reduction in HDRS scores compared to baseline and 2 patients had a $48 \%$ reduction in HDRS scores. Among ten responders, nine (90\%) had MDD and one (10\%) had BD. Seven (70\%) had MDD and three $(30 \%)$ had $\mathrm{BD}$ of the ten non-responders. The response rates in the SPW group were $45.5 \%$ (5 out of 11 ) and LPW were $55.6 \%$ 
a)
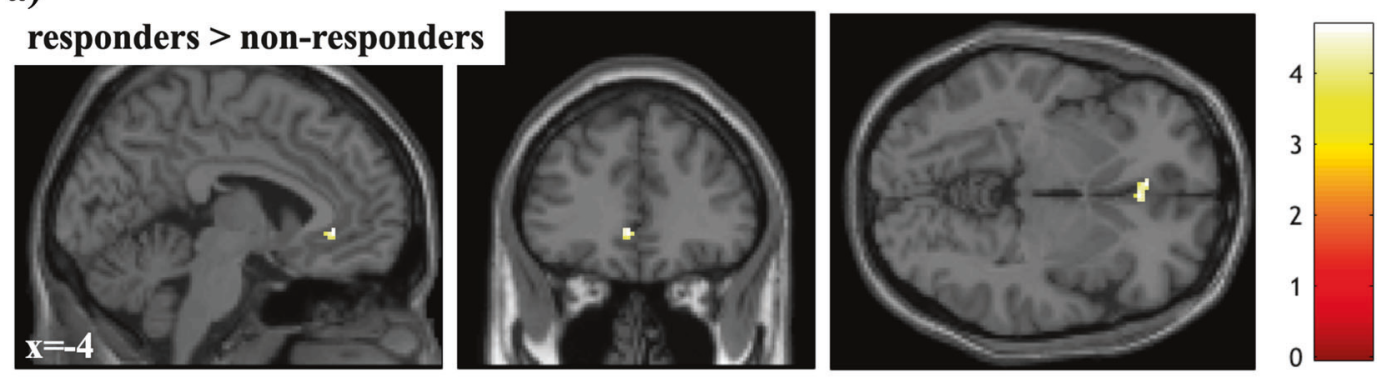

b)

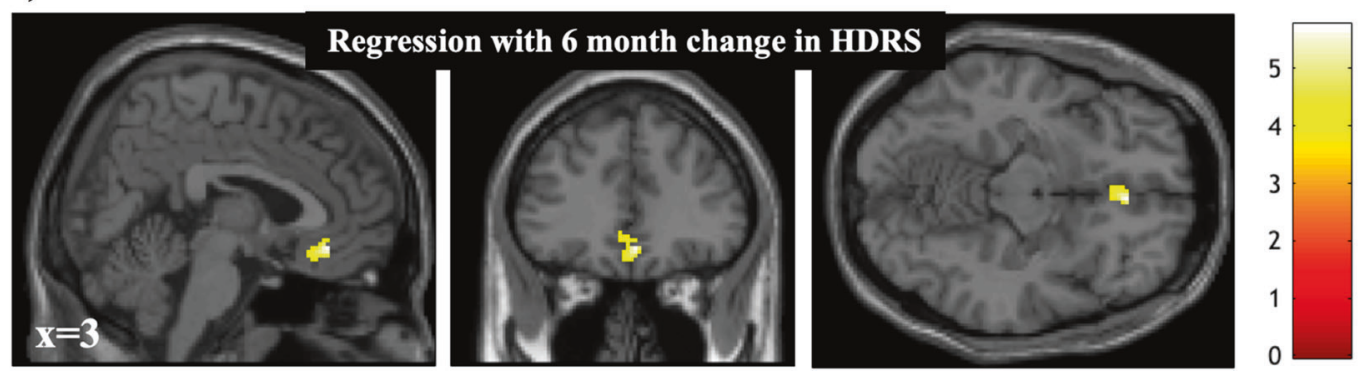

Fig. 2 Treatment prediction results with baseline FDG-PET. a Contrast showing DBS responders had greater baseline FDG-PET metabolic activity (CMRGlu) within the SCC ROI compared to non-responders. b Voxel-wise multiple regression showing a greater reduction in symptom severity was associated with greater baseline CMRGlu. All results thresholded to FWE corrected $p<0.05$ after small volume correction with the SCC ROI, and after controlling for age. HDRS Hamilton Rating Scale for Depression.

(5 out of 9). However, the responders $(n=10)$ were equally distributed between SPW (50\%) and LPW (50\%) stimulation groups. Among ten non-responders, six (60\%) were from SPW group and four (40\%) were from LPW (Supplementary Table S1). The mean percentage change in HDRS was 41.3 ( $\mathrm{SD}=28.2$ ). Table 1 shows the results of statistical tests comparing clinical and demographic data in responders and non-responders. HDRS scores were significantly different at 6 months between responders and non- responders $(t=5.31 ; p<0.001)$, whereas baseline scores between groups were not different $(t=-0.45 ; p=0.66)$. We saw a significant difference in age between responders and non-responders $(t(18)=2.55 ; p=0.02)$, where responders tended to be younger than non-responders. Importantly, there were no differences between responders and non-responders in baseline HDRS scores, duration of illness, duration of current episode and number of antidepressants taken at time of implantation. In addition, there were no apparent group differences in comorbidities (Supplementary Table S1).

Treatment prediction imaging results

Notably, there was no significant relationship between baseline CMRGlu and baseline HDRS scores (after $p<0.05$ FWE correction) with both voxel-wise and mean SCC PET values. However, voxelwise analyses within the SCC ROI comparing responders and nonresponders showed that responders exhibited significantly greater baseline SCC CMRGlu than non-responders after controlling for age (voxels =36; peak voxel: $x=-4, y=36, z=-4$; effect size $(T)=$ 4.69) (Fig. 2a). The voxel-wise multiple regression within the SCC ROI across all patients showed a significant positive relationship between HDRS percentage change (i.e., reduction) at 6 months and baseline SCC CMRGlu, with age included as a regressor of no interest (voxels $=145$; peak voxel: $x=4, y=36, z=-12$; effect size $(T)=5.76$ ) (Fig. 2b). A scatterplot showing baseline SCC CMRGlu as a predictor of treatment response is also presented in the Supplementary Results (Supplementary Fig. S1), where patients diagnosed with bipolar depression are highlighted. In addition, we compared SCC CMRGlu between responders and non-responders after excluding the four bipolar patients. Our results showed only little change when the bipolar patients were excluded (voxels $=38$; peak voxel: $x=0, y=36, z=-4$; effect size $(T)=4.93)$. This demonstrated that baseline SCC metabolic activity was predictive of clinical response in this sample regardless of the type of depression.

Using leave-one-out cross-validation with the naïve Bayes classifier, the baseline mean FDG-PET signal intensity from the SCC ROI could predict which patients responded to treatment with an accuracy of $80 \%$. More precisely, 16 out of the 20 patients were correctly classified; whereas, the incorrectly classified cases were evenly distributed among the two groups with two out of ten responders and two out of ten non-responders being incorrectly classified, leading to an overall sensitivity of $80 \%$ and specificity of $80 \%$.

Treatment marker imaging results

Results of the voxel-wise rmANCOVA within the SCC ROI showed that both responders (voxels $=320$; peak voxel: $x=-6, y=30$, $z=-8$; effect size $(T)=5.07)$ and non-responders (Cluster 1: voxels $=53$; peak voxel: $x=-14, y=14, z=-24$; effect size $(T)=$ 6.27; Cluster 2: voxels $=38$; peak voxel: $x=0, y=36, z=-24$; effect size $(T)=5.21$ ) exhibited a significant reduction in metabolic activity from baseline to 6 months postoperatively (Fig. 3), after controlling for age. Notably, the cluster size representing the difference between baseline and 6 months was substantially larger in responders than non-responders (see Fig. 3). Full-factorial analysis using time as one factor (baseline and 6 months post DBS scan) and group (responders/non-responders) as another factor, including age as a covariate, showed no significant voxels. Furthermore, $t$-test, contrasting 6-month scans for responders vs. non-responders also showed no significant voxels. When controlling for age, the partial correlation between HDRS 6-month percentage change and 6-month percentage change in mean CMRGlu within the SCC ROI showed a significant positive relationship $(r(17)=0.509 ; p=0.031)$ in which a greater change in SCC metabolic activity was associated with a greater change in symptom severity (Fig. 4). Importantly, we saw no significant 
a)
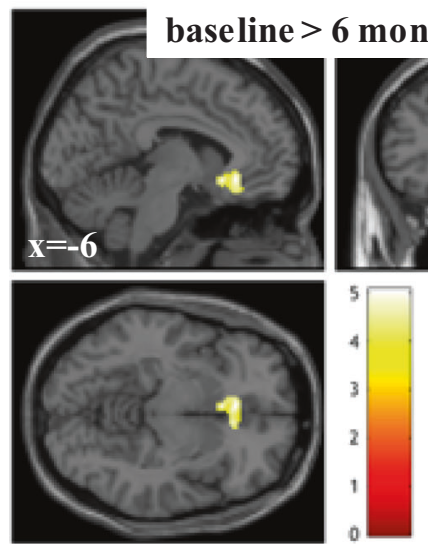

Responders b)
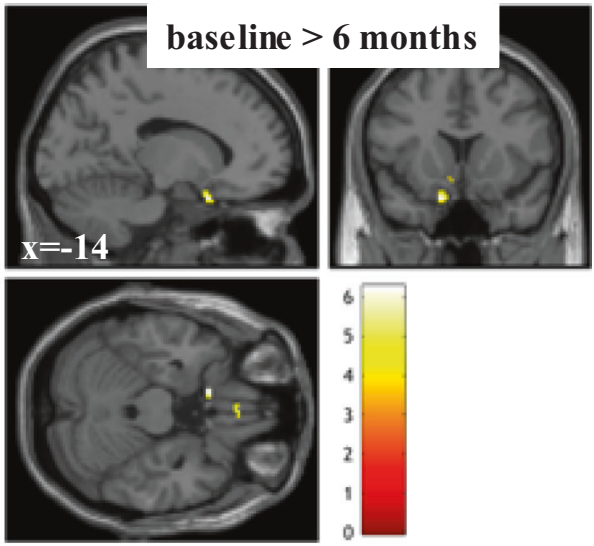

Non-responders

Fig. 3 Treatment marker results in responders and non-responders. Metabolic activity at baseline is greater than after 6 months of DBS of the subcallosal cingulate (SCC) in (a) responders and (b) non-responders. All results thresholded to FWE corrected $p<0.05$ after small volume correction with the SCC ROI.

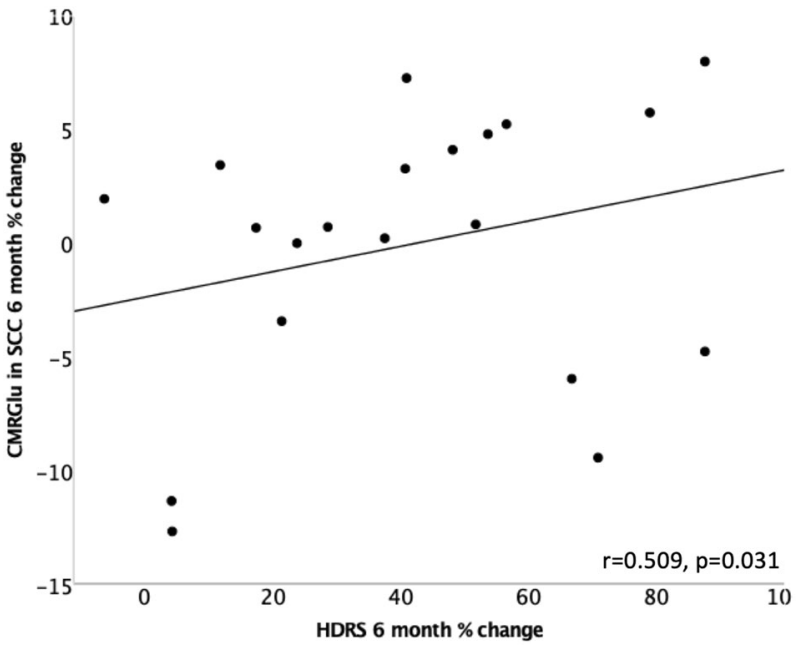

Fig. 4 Treatment marker results in entire sample-scatterplot demonstrating a positive relationship between percentage change in mean metabolic activity (CMRGlu) in the subcallosal cingulate (SCC) and percentage change in symptom severity after 6 months of DBS. Here a greater reduction in symptoms is associated with greater change in SCC CMRGlu ( $r$ and $p$ values corrected for age). HDRS Hamilton Rating Scale for Depression.

correlations between duration of illness and 6-month change in CMRGlu in SCC, and duration of current episode and 6-month change in CMRGlu in SCC. Therefore it was unlikely that CMRGlu differences between response groups were accounted for by illness chronicity.

Whole-brain imaging results

After correction for multiple comparisons (FWE corrected $p<0.05$ ), no significant differences were found between responders and non-responders at the whole-brain level, and no significant relationship between baseline CMRGlu and symptom change. Although interestingly, when investigating the uncorrected results ( $p_{\text {uncorrected }}<0.05$ at the cluster level, $p_{\text {uncorrected }}<0.001$ at the peak level), a positive relationship between symptom change and CMRGlu can be seen in the SCC and insula (Supplementary Fig. 1). Whole-brain treatment marker results, comparing metabolic activity at baseline and 6 months postoperatively, revealed some significant differences when comparing responders and nonresponders that survived corrections for multiple comparisons
(Supplementary Fig. 2). More precisely, responders demonstrated more localized decreases in metabolic activity within the SCC, whereas non-responders showed a decrease in more ventral and anterior areas including ventromedial prefrontal cortex (VMPFC), rostral anterior cingulate (rostral ACC) and OFC. More details on whole-brain results are provided in the Supplementary Results (Supplementary Figs. S2 and S3).

\section{DISCUSSION}

The aim of this study was first to assess whether pre-treatment metabolic activity in the SCC is predictive of DBS treatment outcome at 6 months, and second, whether change in metabolic activity could serve as a treatment marker that tracks clinical improvement over 6 months of chronic DBS. To our knowledge, this is the largest PET imaging study in patients receiving SCC DBS for TRD, and the first to provide evidence for a neuroimaging biomarker of SCC DBS treatment response prediction. Glucose utilization in SCC predicted response, where greater baseline SCC metabolic activity was associated with a greater change in symptom severity at 6 months postoperatively. Furthermore, mean CMRGlu in the SCC could correctly classify responders and non-responders with an accuracy of $80 \%$, providing strong evidence for the use of SCC CMRGlu as a candidate biomarker for SCC DBS treatment outcome. Further evidence of this imagebased treatment biomarker was provided by the greater change in SCC metabolic activity as a result of chronic DBS being associated with greater clinical improvement. Importantly, these results were not confounded by age, illness chronicity or volumetric differences in the SCC. Therefore, SCC CMRGlu has potential for predicting outcome in future SCC DBS for TRD studies with relatively high accuracy. Such a method to select patients prospectively for SCC DBS has substantial clinical significance. By predicting the probability of response at the individual level using an objective method, response rates may improve for this therapy [40].

Studies using FDG-PET to predict pharmacological and psychotherapy treatment outcome have generally reported that responders exhibited lower pre-treatment activity in the SCC. In particular, one study showed that responders to both venlafaxine and cognitive behavioural therapy (CBT) had lower metabolism in SCC when compared to non-responders [41]. Later McGrath et al. also demonstrated that patients who remitted to both escitalopram and CBT had lower pre-treatment SCC metabolic activity than non-responders [42]. Interestingly, our results in SCC DBS patients showed an opposite pattern, with responders having 
greater pre-treatment metabolic SCC activity. However, when we look to studies using other somatic treatments, patterns that are similar to our results are observed. For example, one study using anterior cingulotomy to treat patients with TRD showed that higher pre-treatment metabolic SCC rates were correlated with improvements in depression symptom severity [10]. More recently, greater response to rTMS treatment was also found to be associated with higher baseline SCC metabolic activity [43]. One likely explanation for these conflicting results between antidepressant and somatic treatment studies is the difference in cohorts. The studies that used rTMS and cingulotomy both recruited patients that were highly treatment resistant. Likewise, our patients were highly treatment resistant and may represent a cohort similar to the non-responders included in previous medication and psychotherapy studies. Thus our patients, being non-responders to medication, would exhibit greater SCC metabolic activity relative to responders. In other words, metabolic SCC activity could relate to resistance to different types of treatment, as suggested by Dunlop and Mayberg [44], with patients with the highest levels of SCC metabolism potentially representing a group that is also highly resistant to medication.

DBS can induce local effects on the target and distant effects on connected brain regions with the net result of DBS on neuronal activity being activation, inhibition or both [45]. In this study, inhibitory effects of chronic DBS on the target (SCC) were found, consistent with two previous studies that showed decreases in SCC CBF in TRD patients that received SCC DBS [1, 22]. However, unlike another SCC DBS study [17], we did not find an increase in metabolic activity within the SCC white matter. According to findings that the metabolic activity of white matter is 2-4 times lower in grey matter [46], changes in metabolism in white matter may have been masked by signal-to-noise ratio.

The SCC DBS-induced inhibition of the SCC target seen in our study may reflect an underlying mechanism of the antidepressant effect, specifically because the decrease in SCC metabolic activity was related to a reduction in depressive symptoms. Taking into account that SCC activity can predict as well as monitor clinical responses to DBS, it therefore has potential not only as an imagebased biomarker for patient selection, but also as a surrogate marker of clinical improvement. Besides the local effects seen in responders, we also found a decrease in metabolic activity in adjacent areas including VMPFC, OFC and rostral ACC in nonresponders, which is similar to previous PET findings in SCC DBS $[1,17]$. The DBS-induced decrease in SCC metabolic activity and adjacent areas may be explained by activation of afferent and efferent axons and the inhibition of cell bodies during DBS, as previous DBS studies have suggested [45]. This is also in line with a preclinical study showing synaptic depression in motor cortex with subcortical white-matter DBS [47], although more complex cellular mechanisms may also be involved in prefrontal regions.

Contrary to some previous SCC DBS studies [1, 17], we did not find distant effects in hypothalamus, dorsolateral prefrontal cortex, posterior cingulate or parietal regions even though we used white-matter-based tractography to target these tracts emanating from the SCC region. While we do not know the exact reasons for the lack of distant effects, several reasons are possible. The differences in methodologies between our study and previous studies in terms of patient selection, sample size, electrode placement, PET imaging acquisition, stimulation protocols and statistical analysis might all have contributed to the discrepancies in the results. First, depression is a heterogenous condition with individual variations in symptoms and neural circuit abnormalities [48]. In addition, our study included patients with bipolar depression. The variations in neural mechanisms underlying disease pathology and treatment recovery within the subtypes of unipolar depression and between unipolar and bipolar depression $[49,50]$ might have contributed to the disparity. Second, it is also important to note that previous PET results were derived from small sample sizes and were not statistically corrected for multiple comparisons, so that the distal effects observed in previous studies may be representative of smaller effects. Third, LPW (210-450 $\mu$ s) optimization was used in half of the patients studied, whereas previous studies used only SPW optimization [30]. Contrary to short pulses, LPW stimulation may also affect smaller axons, which often project more locally [51]. Fourth, DBS may induce normalization of a distal network through the local effects (i.e., inhibition of SCC and adjacent sites) without apparent increases in the activation of the distal regions, as these brain regions are well integrated and are interrelated networks responsible for automatic and voluntary emotional regulation [52]. This is partly supported by a recent SCC DBS discontinuation study in which stable responders showed decreases in dACC and a premotor region when DBS was switched off compared to the preDBS baseline, whereas there were no increases in dACC and the premotor region when DBS was turned on, relative to baseline [29]. Since our study design did not include on and off stimulation conditions, we can only speculate about these mechanisms.

The response rate in this sample was comparable to other open-label trials, but was also considerably higher than a recent large multicentre sham-controlled trial (17\%) [7]. There are several possible reasons for this, which could include differences in patient characteristics, the use of aggressive high current density stimulation, and DTI-based precision targeting in our study. Our study was conducted in a single centre, whereas the shamcontrolled trial was multicenter. In multicenter studies, it is difficult to standardize surgical procedures and targeting across the centres. Results of single centre studies have been shown to be better than multicenter studies [3, 17]. Furthermore, the impact of uncertainty in patients and researchers in a sham-controlled study (i.e., whether they are receiving stimulation or not) would be different compared to our study, which uses an active control group, where stimulation is certain (i.e., only uncertainty of whether treatment results will be different between two stimulation types).

There are limitations of this study that should be discussed. Our sample size was small, relative to more general neuroimaging studies, especially for between-group analysis comparing responders and non-responders. Therefore our findings should be considered preliminary until replicated or prospectively validated in a larger sample. As we were only able to collect FDG-PET in the DBS on state, we cannot fully determine whether the change in metabolic activity over time was caused by stimulation, or other variables related to the surgical procedure, such as microlesioning. However, micro-lesioning effects typically wear off after a few days to weeks $[53,54]$, and therefore were unlikely to have had substantial effects after 6 months. Our study design did not include a sham-control group and therefore it was not possible to tease out effects of potential placebo-induced recovery. Another limitation was that we did not have a healthy control group so it was not possible to determine whether changes in SCC metabolic activity of the SCC and other regions represented a normalization of activity. We were also not able to control for possible confounding effects of medication on metabolic activity, as all patients were taking various medications at the time of scanning. However, medications remained mostly unchanged during the first 6 months of DBS except in one non-responder. There was also no difference in the number or type of medications between responders and non-responders. The high cost of PET scanning may be a barrier for clinical translation. However, future use of PET guided patient selection for DBS surgery would be more cost effective overall because resources could be focused on patients who have a high chance of responding. The heterogeneity in the sample composition with MDD and BD patients may complicate the interpretation of our findings. However, only $20 \%$ of patients in our sample had bipolar depression, and therefore our findings were predominantly driven by MDD patients. To confirm this 
point, we compared SCC CMRGlu between responders and nonresponders after excluding four bipolar patients. Our results showed little change when the bipolar patients were excluded. Furthermore, converging evidence from structural and functional imaging studies suggest that both MDD and BD share a common underlying aberrant circuitry involving SCC [55] and it is therefore possible that there may be similarities in SCC DBS treatment changes in BD and MDD. Due to our small sample size, we could not perform subgroup analysis. Future SCC DBS studies should examine the similarities and/or differences in prediction and treatment changes in SCC between MDD and BD using larger sample sizes.

In conclusion, we present strong evidence that SCC metabolic activity could serve as a potential image-based biomarker to predict treatment outcome of DBS in patients with depression. The change in metabolic activity following chronic DBS was related to clinical improvement, implying its potential as an objective surrogate treatment marker for future DBS trials. Predicting the efficacy of treatments at the individual level is one of the most important goals for clinical research that could have the most potential to translate to the clinic. Here we show that SCC metabolic activity could serve as a predictor to help select patients most likely to respond to SCC DBS. Although a prospective study is needed to confirm that this biomarker is indeed helpful for patient selection. As DBS procedures are costly, invasive and not without risk, future DBS trials should be personalized using baseline SCC metabolic activity in addition to optimizing stimulation of SCC-fibre tracts to ultimately improve response rates.

\section{FUNDING AND DISCLOSURE}

Funding was provided by Alberta Innovates Health Solutions (AIHS). ZHTK was an AlHS Clinical Scholar, and ECB and DLC were both AlHS-funded post-doctoral fellows. RR has received honorarium for serving in the advisory committee of Astra Zeneca, Lundbeck, Janssen and Otsuka. He also received an investigatorinitiated grant from Astra Zeneca and Pfizer. ZHTK reports that Alberta Health Services receives value adds for research and education from Medtronic Canada, the manufacturer of the DBS systems used in the study. These funds are only partially available for research and education through the Neuromodulation programme. Medtronic had no involvement in any of this study. Other authors declare no potential competing interests.

\section{ACKNOWLEDGEMENTS}

We thank Dr. Mayberg for her guidance in the development of the PET protocol. We also thank Dr. Yatham Lakshmi and Dr. Vesna Sossi, at the University of British Columbia, for providing training in imaging analysis. This study was presented as a poster at the 73rd Society of Biological Psychiatry annual meeting on May 2018.

\section{AUTHOR CONTRIBUTIONS}

ECB performed the imaging analyses and wrote the paper. RR/ZHTK conceptualized and designed the study of subcallosal cingulate deep brain stimulation for treatmentresistant depression. RR designed the PET study. ZHTK implanted the DBS system. RR performed initial psychiatric assessment and monthly clinical assessments to determine responders and non-responders. NDF performed the machine learning analysis. CPM assisted in developing the PET protocol, and PET image acquisition and storage. RR, ZHTK, ECB and DLC interpreted the results. All authors reviewed the final paper and provided comments.

\section{REFERENCES}

1. Mayberg HS, Lozano AM, Voon V, McNeely HE, Seminowicz D, Hamani C, et al. Deep brain stimulation for treatment-resistant depression. Neuron. 2005;45:651-60.

2. Holtzheimer PE, Kelley ME, Gross RE, Filkowski MM, Garlow SJ, Barrocas A, et al. Subcallosal cingulate deep brain stimulation for treatment-resistant unipolar and bipolar depression. Arch Gen Psychiatry. 2012;69:150-8.
3. Lozano AM, Giacobbe P, Hamani C, Rizvi SJ, Kennedy SH, Kolivakis T, et al. A multicenter pilot study of subcallosal cingulate area deep brain stimulation for treatment-resistant depression. J Neurosurg. 2012;116:315-22.

4. Merkl A, Schneider G-H, Schönecker T, Aust S, Kühl K-P, Kupsch A, et al. Antidepressant effects after short-term and chronic stimulation of the subgenual cingulate gyrus in treatment-resistant depression. Exp Neurol. 2013;249:160-8.

5. Puigdemont D, Pérez-Egea R, Portella MJ, Molet J, de Diego-Adeliño J, Gironell A, et al. Deep brain stimulation of the subcallosal cingulate gyrus: further evidence in treatment-resistant major depression. Int J Neuropsychopharmacol. 2012;15: 121-33.

6. Ramasubbu R, Anderson S, Haffenden A, Chavda S, Kiss ZHT. Double-blind optimization of subcallosal cingulate deep brain stimulation for treatmentresistant depression: a pilot study. J Psychiatry Neurosci. 2013;38:325-32.

7. Holtzheimer PE, Husain MM, Lisanby SH, Taylor SF, Whitworth LA, McClintock S, et al. Subcallosal cingulate deep brain stimulation for treatment-resistant depression: a multisite, randomised, sham-controlled trial. Lancet Psychiatry. 2017;4:839-49.

8. Hamani C, Mayberg H, Stone S, Laxton A, Haber S, Lozano AM. The subcallosal cingulate gyrus in the context of major depression. Biol Psychiatry. 2011;69:301-8.

9. Ramirez-Mahaluf JP, Perramon J, Otal B, Villoslada P, Compte A. Subgenual anterior cingulate cortex controls sadness-induced modulations of cognitive and emotional network hubs. Sci Rep. 2018;8:8566.

10. Dougherty DD, Weiss AP, Cosgrove GR, Alpert NM, Cassem EH, Nierenberg AA, et al. Cerebral metabolic correlates as potential predictors of response to anterior cingulotomy for treatment of major depression. J Neurosurg. 2003;99:1010-7.

11. Drevets WC, Price JL, Simpson JR, Todd RD, Reich T, Vannier M, et al. Subgenual prefrontal cortex abnormalities in mood disorders. Nature. 1997;386:824-7.

12. Mayberg HS, Liotti M, Brannan SK, McGinnis S, Mahurin RK, Jerabek PA, et al. Reciprocal limbic-cortical function and negative mood: converging PET findings in depression and normal sadness. Am J Psychiatry. 1999;156:675-82.

13. Seminowicz DA, Mayberg HS, Mclntosh AR, Goldapple K, Kennedy S, Segal Z, et al. Limbic-frontal circuitry in major depression: a path modeling metanalysis. Neurolmage. 2004;22:409-18.

14. Dunlop BW, Rajendra JK, Craighead WE, Kelley ME, McGrath CL, Choi KS, et al. Functional connectivity of the subcallosal cingulate cortex and differential outcomes to treatment with cognitive-behavioral therapy or antidepressant medication for major depressive disorder. Am J Psychiatry. 2017;174:533-45.

15. Mayberg HS, Brannan SK, Tekell JL, Silva JA, Mahurin RK, McGinnis S, et al. Regional metabolic effects of fluoxetine in major depression: serial changes and relationship to clinical response. Biol Psychiatry. 2000;48:830-43.

16. Liu Y, Du L, Li Y, Liu H, Zhao W, Liu D, et al. Antidepressant effects of electroconvulsive therapy correlate with subgenual anterior cingulate activity and connectivity in depression. Medicine. 2015;94:e2033.

17. Lozano AM, Mayberg HS, Giacobbe P, Hamani C, Craddock RC, Kennedy SH. Subcallosal cingulate gyrus deep brain stimulation for treatment-resistant depression. Biol Psychiatry. 2008;64:461-7.

18. Nobler MS, Oquendo MA, Kegeles LS, Malone KM, Campbell CC, Sackeim HA, et al. Decreased regional brain metabolism after ect. Am J Psychiatry. 2001;158:305-8.

19. Siegle GJ, Thompson WK, Collier A, Berman SR, Feldmiller J, Thase ME, et al. Toward clinically useful neuroimaging in depression treatment: prognostic utility of subgenual cingulate activity for determining depression outcome in cognitive therapy across studies, scanners, and patient characteristics. Arch Gen Psychiatry. 2012;69:913-24.

20. Pizzagalli D, Pascual-Marqui RD, Nitschke JB, Oakes TR, Larson CL, Abercrombie $\mathrm{HC}$, et al. Anterior cingulate activity as a predictor of degree of treatment response in major depression: evidence from brain electrical tomography analysis. Am J Psychiatry. 2001;158:405-15.

21. Pizzagalli DA, Webb CA, Dillon DG, Tenke CE, Kayser J, Goer F, et al. Pretreatment rostral anterior cingulate cortex theta activity in relation to symptom improvement in depression: a randomized clinical trial. JAMA Psychiatry. 2018;75:547-54.

22. Conen S, Matthews JC, Patel NK, Anton-Rodriguez J, Talbot PS. Acute and chronic changes in brain activity with deep brain stimulation for refractory depression. J Psychopharmacol Oxf Engl. 2018;32:430-40.

23. Lipsman N, Lam E, Volpini M, Sutandar K, Twose R, Giacobbe P, et al. Deep brain stimulation of the subcallosal cingulate for treatment-refractory anorexia nervosa: 1 year follow-up of an open-label trial. Lancet Psychiatry. 2017;4:285-94.

24. Bewernick BH, Hurlemann R, Matusch A, Kayser S, Grubert C, Hadrysiewicz B, et al. Nucleus accumbens deep brain stimulation decreases ratings of depression and anxiety in treatment-resistant depression. Biol Psychiatry. 2010;67:110-6.

25. Dougherty DD, Chou T, Corse AK, Arulpragasam AR, Widge AS, Cusin C, et al. Acute deep brain stimulation changes in regional cerebral blood flow in obsessive-compulsive disorder. J Neurosurg. 2016;125:1087-93.

26. Le Jeune F, Vérin $M, N^{\prime}$ Diaye $K$, Drapier D, Leray E, Du Montcel ST, et al. Decrease of prefrontal metabolism after subthalamic stimulation in obsessive-compulsive disorder: a positron emission tomography study. Biol Psychiatry. 2010;68:1016-22. 
27. Rauch SL, Dougherty DD, Malone D, Rezai A, Friehs G, Fischman AJ, et al. A functional neuroimaging investigation of deep brain stimulation in patients with obsessive-compulsive disorder. J Neurosurg. 2006;104:558-65.

28. Suetens K, Nuttin B, Gabriëls L, Van Laere K. Differences in metabolic network modulation between capsulotomy and deep-brain stimulation for refractory obsessive-compulsive disorder. J Nucl Med. 2014;55:951-9.

29. Martín-Blanco A, Serra-Blasco M, Pérez-Egea R, de Diego-Adeliño J, CarcellerSindreu $M$, Puigdemont $D$, et al. Immediate cerebral metabolic changes induced by discontinuation of deep brain stimulation of subcallosal cingulate gyrus in treatment-resistant depression. J Affect Disord. 2015;173:159-62.

30. Ramasubbu R, Clark DL, Golding S, Dobson KS, Mackie A, Haffenden A, et al. Long versus short pulse width subcallosal cingulate stimulation for treatment-resistant depression: a randomised, double-blind, crossover trial. Lancet Psychiatry. 2020; 7:29-40.

31. Bewernick BH, Kayser S, Gippert SM, Switala C, Coenen VA, Schlaepfer TE. Deep brain stimulation to the medial forebrain bundle for depression- long-term outcomes and a novel data analysis strategy. Brain Stimul. 2017;10:664-71.

32. Kelley ME, Franco AR, Mayberg HS, Holtzheimer PE. The Illness Density Index (IDI): a longitudinal measure of treatment efficacy. Clin Trials. 2012;9:596-604.

33. van der Wal JM, Bergfeld IO, Lok A, Mantione M, Figee $M$, Notten $P$, et al. Long-term deep brain stimulation of the ventral anterior limb of the internal capsule for treatment-resistant depression. J Neurol Neurosurg Psychiatry. 2020;91:189-95.

34. Makris N, Goldstein JM, Kennedy D, Hodge SM, Caviness VS, Faraone SV, et al. Decreased volume of left and total anterior insular lobule in schizophrenia. Schizophr Res. 2006;83:155-71.

35. Frazier JA, Chiu S, Breeze JL, Makris N, Lange N, Kennedy DN, et al. Structural brain magnetic resonance imaging of limbic and thalamic volumes in pediatric bipolar disorder. Am J Psychiatry. 2005;162:1256-65.

36. Desikan RS, Ségonne F, Fischl B, Quinn BT, Dickerson BC, Blacker D, et al. An automated labeling system for subdividing the human cerebral cortex on MRI scans into gyral based regions of interest. Neurolmage. 2006;31:968-80.

37. Goldstein JM, Seidman LJ, Makris N, Ahern T, O'Brien LM, Caviness VS, et al. Hypothalamic abnormalities in schizophrenia: sex effects and genetic vulnerability. Biol Psychiatry. 2007;61:935-45.

38. Brett M, Anton J-L, Valabregue R, Poline J-B. Region of interest analysis using SPM toolbox (Abstract). In 8th international conference on Functional Mapping of the Human Brain. Sendai, Japan. Neuroimage. 2002:16.

39. Ashburner J, Friston KJ. Voxel-based morphometry-the methods. Neurolmage. 2000;11:805-21.

40. Bari AA, Mikell CB, Abosch A, Ben-Haim S, Buchanan RJ, Burton AW, et al. Charting the road forward in psychiatric neurosurgery: proceedings of the 2016 American Society for Stereotactic and Functional Neurosurgery workshop on neuromodulation for psychiatric disorders. J Neurol Neurosurg Psychiatry. 2018;89:886-96.

41. Konarski JZ, Kennedy SH, Segal ZV, Lau MA, Bieling PJ, McIntyre RS, et al. Predictors of nonresponse to cognitive behavioural therapy or venlafaxine using glucose metabolism in major depressive disorder. J Psychiatry Neurosci. 2009;34:175-80.
42. McGrath CL, Kelley ME, Dunlop BW, Holtzheimer PE, Craighead WE, Mayberg HS. Pretreatment brain states identify likely nonresponse to standard treatments for depression. Biol Psychiatry. 2014;76:527-35.

43. Baeken C, Marinazzo D, Everaert H, Wu G-R, Van Hove C, Audenaert K, et al. The impact of accelerated HF-rTMS on the subgenual anterior cingulate cortex in refractory unipolar major depression: insights from 18FDG PET brain imaging. Brain Stimul. 2015;8:808-15.

44. Dunlop BW, Mayberg HS. Neuroimaging-based biomarkers for treatment selection in major depressive disorder. Dialogues Clin Neurosci. 2014;16:479-90.

45. McIntyre CC, Grill WM, Sherman DL, Thakor NV. Cellular effects of deep brain stimulation: model-based analysis of activation and inhibition. J Neurophysiol. 2004;91:1457-69.

46. Berti V, Mosconi L, Pupi A. Brain: normal variations and benign findings in fluorodeoxyglucose-PET/computed tomography imaging. PET Clin. 2014;9: 129-40.

47. Iremonger KJ, Anderson TR, Hu B, Kiss ZHT. Cellular mechanisms preventing sustained activation of cortex during subcortical high-frequency stimulation. J Neurophysiol. 2006;96:613-21.

48. Akil H, Gordon J, Hen R, Javitch J, Mayberg H, McEwen B, et al. Treatment resistant depression: a multi-scale, systems biology approach. Neurosci Biobehav Rev. 2018;84:272-88.

49. Drysdale AT, Grosenick L, Downar J, Dunlop K, Mansouri F, Meng Y, et al. Restingstate connectivity biomarkers define neurophysiological subtypes of depression. Nat Med. 2017;23:28-38.

50. Lawrence NS, Williams AM, Surguladze S, Giampietro V, Brammer MJ, Andrew C, et al. Subcortical and ventral prefrontal cortical neural responses to facial expressions distinguish patients with bipolar disorder and major depression. Biol Psychiatry. 2004;55:578-87.

51. Anderson CJ, Anderson DN, Pulst SM, Butson CR, Dorval AD. Neural selectivity, efficiency, and dose equivalence in deep brain stimulation through pulse width tuning and segmented electrodes. BioRxiv. 2019:613133. https://doi.org/10.1101/ 613133.

52. Rive MM, van Rooijen G, Veltman DJ, Phillips ML, Schene AH, Ruhé HG. Neural correlates of dysfunctional emotion regulation in major depressive disorder. A systematic review of neuroimaging studies. Neurosci Biobehav Rev. 2013;37: 2529-53.

53. Cersosimo MG, Raina GB, Benarroch EE, Piedimonte F, Alemán GG, Micheli FE. Micro lesion effect of the globus pallidus internus and outcome with deep brain stimulation in patients with Parkinson disease and dystonia. Mov Disord. 2009;24: 1488-93.

54. Bewernick BH, Kayser S, Sturm V, Schlaepfer TE. Long-term effects of nucleus accumbens deep brain stimulation in treatment-resistant depression: evidence for sustained efficacy. Neuropsychopharmacology. 2012;37:1975-85.

55. Lipsman N, Mclntyre RS, Giacobbe P, Torres C, Kennedy SH, Lozano AM. Neurosurgical treatment of bipolar depression: defining treatment resistance and identifying surgical targets. Bipolar Disord. 2010;12:691-701. 